\title{
CONTROLE DE ESTÍMULOS E CONTRASTE COMPORTAMENTAL EM UMA TAREFA DE COOPERAÇÃO
}

\author{
Rafaela Meireles Fontes AzeVedo \\ JOÃO CLAUDIO TODOROV \\ UNIVERSIDADE DE BRASÍLIA, BRASIL
}

STIMULUS CONTROL AND BEHAVIORAL CONTRAST IN A COOPERATIVE TASK

\begin{abstract}
RESUMO
Metacontingência, definida como um conjunto de contingências comportamentais entrelaçadas que produzem um produto agregado mantido por uma consequência, é uma unidade de análise no nível cultural, análoga à tríplice contingência. Estudos realizados na área têm tratado a metacontingência como uma relação condicional de dois termos, desconsiderando a função dos estímulos antecedentes. Em função da importância dos estímulos discriminativos sobre a emissão do comportamento, e buscando estudar os processos básicos envolvidos, o presente estudo teve como objetivo investigar os efeitos do processo de discriminação em tarefas de cooperação. O procedimento foi realizado a partir de um jogo colaborativo em um tabuleiro de xadrez virtual e teve como produto agregado o encontro dos cavalos. O experimento contou com quatro fases: Linha de Base, Treino de Discriminação, Teste de Discriminação e Teste de Generalização. Participaram do experimento 22 estudantes universitários, distribuídos em 11 duplas. Cada dupla passou pelas quatro fases e participou da atividade apenas uma vez. As medidas observadas foram: taxa de produto agregado ao longo das quatro fases, número de movimentos realizados e índice de dispersão. Os resultados indicam que ocorreu responder diferencial entre os estímulos que sinalizavam as diferentes metacontingências em vigor, embora os dados no Teste de Generalização não tenham sido sistemáticos. Outro efeito observado foi o de contraste comportamental, quando foi introduzida uma condição de extinção na fase de Treino. Os dados relacionados ao número de movimentos e índice de dispersão demonstraram a estereotipia gerada pelo reforçamento e variabilidade induzida pela extinção, ambos efeitos observados no comportamento operante.
\end{abstract}

Palavras-chave: metacontingência, controle de estímulos, discriminação operante, estímulo discriminativo, contraste comportamental

\begin{abstract}
Metacontingency, defined as a set of interlocking behavioral contingencies that produce an aggregate product maintained by a consequence, is a unit of analysis similar to the triple contingency. Studies in this area have treated the metacontingency as a conditional relation of two terms, disregarding the role of antecedent stimuli. Due to the importance of discriminative stimuli on the emission of behavior, and in an attempt to study the basic processes involved, this study aimed to investigate the effects of the discrimination process in metacontingencies. The procedure involved a collaborative game on a virtual chessboard and defined the aggregated product as the meeting of two knights. The experiment consisted of four phases: Baseline, Discrimination Training, Discrimination Test and Generalization Test. Twenty-two college students, distributed in 11 dyads, participated in the experiment. Each dyad participated in the activity only once. The measures were the rate of the aggregate product over the four phases, the number of movements, and dispersion index. The results indicated the occurrence of differential responding to the stimuli correlated with the different metacontingencies (stimulus control) although generalization test data were unsystematic. Behavioral contrast occurred after the introduction of an extinction condition in the training phase. The number of movements and the dispersion index showed the stereotyped pattern generated by the reinforcement, and variability induced by extinction, both effects observed in operant behavior.

Keywords: metacontingency, stimulus control, operant discrimination, discriminative stimulus, behavioral contrast
\end{abstract}

Trabalho baseado na Dissertação de Mestrado de Rafaela Meireles Fontes Azevedo do Programa de Pós-Graduação em Ciências do Comportamento, Departamento de Processos Psicológicos Básicos, Instituto de Psicologia da Universidade de Brasília. João Claudio Todorov é Bolsista de Produtividade Científica 1D do CNPq. E-mail: rafaelafontes@ hotmail.com 
Embora a proposta de uma ciência do comportamento se centre em explicar o nível ontogenético de seleção e como o comportamento do indivíduo se estabelece ao longo da sua história de vida, Skinner (1953/2003) demonstrou também interesse em fenômenos que envolvem mais de um indivíduo atuando no que ele chamou de comportamento social, "definido como o comportamento de duas ou mais pessoas em relação uma à outra ou em conjunto em relação ao ambiente comum" (p. $325)$.

Os estudos sobre cooperação realizados principalmente entre as décadas de 50 e 70 demonstraram o interesse da Análise do Comportamento em dar conta de fenômenos que envolvem ação conjunta de mais de um indivíduo. Entretanto, esses estudos ainda faziam uso da tríplice contingência enquanto unidade de análise e explicavam o comportamento a partir de contingências individuais atuando simultaneamente sobre mais de um indivíduo, ou compreendiam o comportamento de um estabelecendo as contingências para o comportamento do outro, atuando como estímulo discriminativo e reforço (Azrin \& Lindsley, 1956; Hake, Olvera \& Bell, 1975; Schimitt, 1976; Schimitt \& Marwell, 1968, 1971a, 1971b).

Para dar conta dos fenômenos que extrapolam as contingências individuais e que não são abarcados pela tríplice contingência enquanto unidade de análise, tarefas nas quais pelo menos duas pessoas cooperam para a realização de um produto comum têm sido estudadas com o auxílio do conceito de metacontingência (Glenn \& Malott, 2004; Todorov, 2012a, 2012b). Todorov (2012a, 2012b) afirma que na metacontingência o que é selecionado não são as contingências entrelaçadas que caracterizam a cooperação, mas o produto agregado. "Podemos dizer que a consequência seleciona um produto agregado, independentemente de variações nas CCEs. O importante são as características do PA. Potencialmente $\underline{\mathbf{n}}$ diferentes CCEs podem produzir o PA requerido pela metacontingência" (Todorov, 2012a, p. 39).

Algumas tentativas de se estudar o conceito experimentalmente têm sido realizadas (e.g., Todorov, 2013; Vieira, 2010). O que se observa é que frequentemente têm-se manipulado como variável independente alguma relação condicional, a fim de verificar o efeito das consequências nas CCEs e em seus respectivos PAs. Vieira (2010) afirma:

... até o momento, o que tem sido investigado experimentalmente em relação a metacontingências refere-se à contingência entre os entrelaçamentos e seus produtos agregados (CCEs $\rightarrow$ PA) e as consequências culturais. Tal relação é análoga à relação resposta-reforçador na contingência operante, ou a uma contingência básica de dois termos. (pp. 22-23)

Assim, além da complexidade de situações de escolha nos estudos iniciais que utilizam o conceito de metacontingência (cf., Todorov, 2013), outra caraterística notória nesses estudos é o fato de a metacontingência ser tratada de maneira análoga a uma contingência de dois termos. Porém, Skinner (1953/2003) afirma que no estudo do operante os estímulos antecedentes à emissão do comportamento exercem uma função bastante relevante por sinalizarem a presença ou ausência de reforço. Todorov (2012b) afirma que

... no ambiente natural raramente a contingência operante de dois termos (resposta e consequência) é válida para qualquer situação. É mais comum a observação de relações descritas pela contingência de três termos, ou tríplice: "se na situação $\mathrm{S} 1$ o comportamento R1 ocorrer, então a consequência S1 será apresentada". (p. 51)

\section{A função dos estímulos antecedentes}

Um princípio geral que norteia a Análise do Comportamento é a compreensão de que o comportamento operante é controlado por suas consequências. Um segundo princípio geral é que o ambiente passa a controlar o comportamento a partir da relação que estabelece com as consequências reforçadoras (Sidman, 2008). Assim, o comportamento operante deve ser descrito tanto em termos de sua relação com a consequência que o seleciona, quanto de sua relação com os estímulos antecedentes presentes na ocasião em que houve apresentação do reforço (Sério, Andery, Gioia, \& Micheletto, 2002; Todorov, 2002).

O processo de discriminação se refere, então, ao estabelecimento de uma classe de respostas que ocorre na presença de uma determinada classe de estímulos $\left(S^{D}\right.$ ou $\mathrm{S}+$ ), que sinaliza a presença do estímulo reforçador, e não ocorre (ou ocorre em menor frequência) na ausência desse estímulo ou na presença de um estímulo que sinalize a ausência do reforço ( $\mathrm{S}^{\Delta}$ ou $\mathrm{S}$-).

Nos estudos iniciais um procedimento utilizado para mensurar o controle de estímulos envolvia testes em extinção, em que os estímulos antecedentes eram apresentados sem que houvesse consequências diferenciais entre eles, e era, então, observada a taxa de respostas emitida na presença de cada estímulo (Dinsmoor, 1950; Morse \& Skinner, 1958). Outra maneira de se avaliar o controle de estímulos é a partir de testes de generalização, que se caracterizam pela apresentação de uma série de estímulos semelhantes aos estímulos treinados, sem que nenhum deles esteja relacionado a alguma consequência, e observa-se como a taxa de resposta se distribui ao longo do contínuo, tendo-se, tipicamente, o pico de respostas durante a apresentação do estímulo correlacionado ao reforço durante o treino (Honig \& Urcuioli, 1981).

Dois efeitos geralmente observados em treinos de discriminação são o deslocamento do pico do gradiente de generalização e o efeito de contraste comportamental. O deslocamento do pico se refere a um afastamento do pico de respostas do $\mathrm{S}+$ na direção oposta ao $\mathrm{S}$ - (Terrace, 1966). O efeito de contraste se refere à alteração da taxa de respostas durante a apresentação de um estímulo, em função de alterações feitas no esquema de reforçamento associado a um estímulo diferente, sendo essa mudança na taxa de respostas durante o primeiro estímulo na direção oposta à taxa produzida pelo estímulo no qual o esquema 
foi alterado (Reynolds, 1961a). O contraste pode ser positivo - quando em um esquema múltiplo se observa um aumento da taxa de respostas no componente que é mantido constante em função de uma "piora" nas condições de reforçamento do componente que é alterado, ou negativo - quando a taxa de respostas no componente constante diminui em função de uma "melhora" nas condições de reforçamento do componente alterado (McSweeney, Ettinger, \& Norman, 1981). O contraste ocorre tipicamente, mas não apenas, durante a formação de discriminação entre dois estímulos, sendo um deles correlacionado com a ausência de reforço.

\section{Controle de estímulos em contextos sociais}

Assim como os estímulos presentes no ambiente físico (inanimado) atuam no controle do comportamento, Skinner (1953/2003) afirma que "um estímulo social, como qualquer outro estímulo, torna-se importante no controle do comportamento por causa das contingências em que se encaixa" (p. 330). Skinner (1953/2003) argumenta a relevância do controle exercido pelo comportamento de outra pessoa em situações de cooperação, que requerem ação combinada dos dois indivíduos, sendo assim necessário que o comportamento de um fique sob controle do comportamento do outro.

Alguns estudos sobre cooperação com humanos investigaram o papel do controle discriminativo na coordenação dos comportamentos dos indivíduos (Schmitt, \& Marwell, 1968). Porém, o tipo de controle de estímulos manipulado nesses estudos, em analogia à metacontingência, se refere ao controle de estímulo que ocorre ainda no nível do entrelaçamento, ou seja, o comportamento de um atuando como $\mathrm{S}^{\mathrm{D}}$ para o comportamento do outro (nível operante). Considerando que a metacontingência é utilizada para analisar o processo de seleção do produto agregado (Todorov, 2012a), ainda faltam estudos que demonstrem a função exercida por estímulos antecedentes que sinalizam o reforço do produto agregado (que é diferente dos estímulos providos pelos comportamentos entrelaçados dos indivíduos envolvidos). O único estudo conhecido até o momento, que trabalhou com esse tipo de manipulação, é o da Vieira (2010), que investigou o papel que estímulos antecedentes podem exercer na metacontingência.

O experimento de Vieira (2010) segue a tradição de experimentos sobre metacontingências, utilizando o procedimento de Pereira (2008), que trabalhou com a inserção coordenada de números, manipulando consequências individuais e culturais e troca de gerações. Os estímulos antecedentes manipulados eram a cor de fundo da tela do programa: azul e vermelho eram correlacionados com diferentes requisitos para o produto agregado e a liberação da consequência. Os resultados indicaram estabelecimento de controle discriminativo, porém, não se observou sistematicidade nos dados de generalização.

Soares, Cabral, Leite e Tourinho (2014) também realizaram um experimento que envolvia controle de estímulos, embora não envolvesse a manipulação de estímulos antecedentes e treino discriminativo, como no trabalho de Vieira (2010). Soares et al. (2014) trabalharam com diferentes exigências de produto agregado (e entrelaçamento) que demandavam, assim, a discriminação, pelos participantes, dos estímulos que cumpriam ou não as exigências de cada metacontingência em vigor. Utilizando o procedimento de matriz, semelhante ao de Vichi (2004), os autores visaram verificar o estabelecimento de diferentes padrões de entrelaçamento e formação de produto agregado em um delineamento de reversão que manipulava tanto consequências individuais quanto culturais. Os autores observaram que houve "a seleção de duas práticas culturais distintas" (Soares et al., 2014, p. 44), porém, os dados não são sistemáticos. Embora tenham sido demonstradas tanto a seleção operante, quanto a seleção cultural, a partir do uso de diferentes consequências, não há evidências de que tenha ocorrido o processo de discriminação das exigências em vigor.

Em função da importância dos estímulos discriminativos sobre a emissão do comportamento e na tentativa de estudar os processos básicos envolvidos no terceiro nível de seleção, de maneira análoga ao estudo do operante, o presente estudo teve como objetivo investigar os efeitos do processo de discriminação simples na metacontingência. Buscou-se analisar se o terceiro nível de seleção respeita os mesmos padrões observados nos estudos de discriminação operante, que se referem ao segundo nível de seleção, a partir de um procedimento de discriminação sucessiva. Este esforço se justifica pelo pequeno número de trabalhos que exploram o papel dos estímulos discriminativos na metacontingência, apesar da relevância já demonstrada destes estímulos no comportamento operante.

Foi utilizado o mesmo procedimento empregado por Vasconcelos \& Todorov (2015). Este procedimento apresenta a vantagem de ser um procedimento simplificado, que respeita os critérios de uma metacontingência, e possibilita um maior controle experimental, tendo em vista que são manipuladas as variáveis apenas no nível cultural, sem a manipulação de contingências operantes atuando no nível individual. $\mathrm{O}$ experimento teve quatro fases: Linha de Base, Treino de Discriminação, Teste de Discriminação e Teste de Generalização. Com esse estudo se pretendeu: 1) testar um procedimento que faz uso da metacontingência enquanto unidade de análise, no qual, diferentemente dos estudos que vem sendo realizados na área, os tipos de consequência (individual ou cultural) não foram manipulados, não havendo consequências programadas para respostas individuais; 2) testar a viabilidade de um treino de discriminação a partir unicamente de reforço social; 3) aplicar o procedimento de discriminação sobre o responder de mais de um indivíduo simultaneamente e; 4) observar os efeitos do treino de discriminação em um teste de generalização.

Foram manipulados os estímulos antecedentes relacionados à presença $\left(\mathrm{S}^{\mathrm{D}}\right)$ ou ausência de reforço $\left(\mathrm{S}^{\Delta}\right)$, a fim de que fosse observado o seu efeito sobre a formação do produto agregado. 


\section{MÉTODO}

\section{Participantes}

Participaram da pesquisa 22 adultos entre 18 e 29 anos (média de 20 anos), estudantes de graduação e de pós-graduação da Universidade de Brasília. Os participantes foram selecionados por conveniência. Ao todo, 11 duplas participaram do experimento, porém, apenas os dados de 8 duplas foram analisados. Três delas foram descartadas da análise de dados por problemas durante o procedimento ${ }^{1}$.

$\mathrm{O}$ projeto foi submetido ao $\mathrm{CEP} / \mathrm{IH}$ Brasil para análise.

\section{Materiais e instrumentos}

A coleta de dados foi realizada no Laboratório de Pesquisa em Avaliação e Medidas (LABPAM), localizado no Instituto de Psicologia da Universidade de Brasília, onde se encontrava uma mesa grande com um notebook, duas cadeiras (dispostas lado a lado na lateral da mesa) e, em separado, uma cadeira para a experimentadora. A experimentadora esteve presente em todas as sessões e, após a entrega das instruções, se mantinha sentada numa cadeira na lateral da mesa onde se encontravam os participantes.

Para a realização da atividade foi utilizado um notebook Samsung, processador Core i3, 2.10GHz, 2GB de RAM, com sistema Microsoft Windows 7 Home Basic. O notebook foi equipado com o software "Xadrez" (Todorov \& Vianney, 2013), que permitia a movimentação das peças por um tabuleiro virtual de $8 \times 8$ células.

O software foi programado com parâmetros específicos em quatro fases. Os resultados gerados pelo software foram: o número de tentativas, acertos, erros, número de movimentos, local de ocorrência de cada movimento, duração de cada movimento, duração de cada apresentação de estímulo e o tempo decorrido em cada fase.

\section{Procedimento}

Cada dupla participou da atividade uma única vez. Ao entrarem na sala de coleta, os participantes receberam e assinaram o TCLE. Em seguida, foi entregue uma ficha para a dupla com instruções sobre a atividade. Não foram apresentadas instruções verbais adicionais e em caso de dúvidas os participantes eram instruídos a ler a ficha novamente. Após a compreensão das instruções, os participantes davam início à atividade.

A atividade consistia na movimentação de duas peças por tabuleiro virtual, sendo cada participante responsável pelo movimento de uma das peças e cada peça se movimentava em L, como o cavalo no xadrez. Cada rodada iniciava com cada uma das peças em um dos

${ }^{1}$ A exclusão das duplas da análise de dados se deveu a motivos como: travamento do programa durante a execução do experimento; desistência de um dos participantes, deixando apenas um responsável por executar a tarefa da dupla; ou desistência de ambos os participantes, que pararam de responder e "aguardaram" o fim da sessão. extremos do tabuleiro e o objetivo era que as peças ficassem em casas adjacentes (horizontal, vertical ou diagonalmente) e isso caracterizava um encontro.

$\mathrm{O}$ experimento teve quatro fases, descritas a seguir.

Linha de Base. Nesta fase o encontro das peças podia ocorrer em qualquer ponto do tabuleiro e todos os encontros eram reforçados com a apresentação da mensagem :

"PARABÉNS! O objetivo foi alcançado!".

A fase se encerrava após 10 encontros. A cor da tela permanecia azul durante toda a fase. Esta primeira fase foi utilizada como base de comparação para as fases subsequentes de treino e teste de discriminação.

Treino de Discriminação. Nessa fase foi realizado um treino discriminativo, no qual foram manipulados os estímulos antecedentes contingentes à disponibilidade do reforço, em um procedimento de discriminação sucessiva. As cores de fundo do tabuleiro, azul ou amarelo, funcionavam como $S^{D}$ e $S^{\Delta}$. A cor de fundo azul funcionava como $\mathrm{S}^{\mathrm{D}}$ (comprimento de onda da cor: $484 \mathrm{~nm}$ ), ou seja, os encontros das peças em qualquer ponto do tabuleiro ocorridos na presença da cor azul eram seguidos de reforço (mensagem "PARABÉNS! O objetivo foi alcançado!'”). A cor de fundo amarela funcionava como $\mathrm{S}^{\Delta}$ (comprimento de onda da cor: $582 \mathrm{~nm}$ ), ou seja, aqui o encontro não gerava consequência (a mensagem de parabéns) e após sua ocorrência as peças voltavam às suas posições iniciais.

A cada tentativa completada na presença do $S^{\mathrm{D}}$, a cor da tela mudava para $\mathrm{S}^{\Delta}$. O critério para encerramento da apresentação do $S^{\Delta}$ se dava por tempo de inatividade, sendo considerado como inatividade um intervalo de tempo sem produção de produto agregado (ou seja, sem ocorrência de encontro), porém, o movimento das peças era permitido. $O$ critério inicial era de 10 segundos na ausência de produto agregado que progredia de 5 em 5 segundos, até que os participantes permanecessem sem se encontrar durante 60 segundos consecutivos. A cada cumprimento do tempo de inatividade exigido durante o $S^{\Delta}$, o tabuleiro voltava para a condição do $S^{D}$, e assim a apresentação dos estímulos se deu de maneira alternada.

Foi utilizada a progressão do tempo, pelo alto número de desistência quando as primeiras exposições à extinção eram muito longas.

A fase se encerrava quando os participantes permaneciam 60 segundos inativos durante 3 apresentações do $S^{\Delta}$. A contagem do tempo era reiniciada sempre que as peças se encontravam na condição do $\mathrm{S}^{\Delta}$.

Teste de Discriminação. Nessa fase foi realizado um teste discriminativo. O procedimento era semelhante ao do Treino de Discriminação, exceto que $\mathrm{S}^{\mathrm{D}}$ e $\mathrm{S}^{\Delta}$ eram apresentados sucessivamente, durante intervalos de 60 segundos.

Nesta fase, os encontros ocorridos na presença do $\mathrm{S}^{\mathrm{D}}$ continuavam a ser seguidos de reforço, enquanto os encontros ocorridos na presença do $\mathrm{S}^{\Delta}$ continuavam em 
extinção. A manutenção do reforço na condição $S^{D}$ foi realizada para evitar que a ocorrência do produto agregado entrasse em extinção e impossibilitasse, assim, a realização do teste de generalização.

A fase era encerrada após 10 minutos (5 apresentações alternadas de cada estímulo) e se caracterizava enquanto teste pela alteração do critério de duração da apresentação dos estímulos em relação à fase anterior. Nesta fase o $S^{\mathrm{D}}$ se mantinha em vigor por 1 minuto e não mais até a ocorrência de um encontro. O $\mathrm{S}^{\Delta}$ também se mantinha em vigor pelo mesmo período de tempo, não sendo mais atrasado pela ocorrência de encontros.

Teste de Generalização. Essa fase era caracterizada pela apresentação de 7 cores. Além das cores que sinalizavam $S^{D}$ e $S^{\Delta}$, outras cinco cores foram utilizadas com os seguintes comprimentos de onda: $566 \mathrm{~nm}, 533 \mathrm{~nm}, 500 \mathrm{~nm}, 468 \mathrm{~nm}$ e $451 \mathrm{~nm}$. Não havia consequências para o responder durante a apresentação de nenhuma das cores, se dando toda a fase em extinção. As cores foram apresentadas uma única vez, durante intervalos de 2 minutos, e foram apresentadas na seguinte ordem: $582 \mathrm{~nm}\left(\mathrm{~S}^{\Delta}\right), 566 \mathrm{~nm}, 533 \mathrm{~nm}, 500 \mathrm{~nm}$, $484 \mathrm{~nm}\left(\mathrm{~S}^{\mathrm{D}}\right), 468 \mathrm{~nm}$ e $451 \mathrm{~nm}$.

Após o fim do Teste de Generalização o programa apresentava a mensagem "Fim!", indicando o término do experimento.

\section{Análise de Dados}

$\mathrm{O}$ que se considerou enquanto resposta no presente trabalho foi $\mathrm{o}$ encontro entre as peças no tabuleiro, que caracterizava a formação do Produto Agregado (PA). Na análise dos dados a medida principal utilizada neste experimento foi a taxa de produto agregado (PA/minuto) em cada uma das fases, ao longo de toda a sessão experimental. Outras medidas observadas em cada fase foram: número de encontros realizados em cada condição e número de movimentos realizados em cada tentativa.

O cálculo da taxa de produto agregado e do número de movimentos foi feito por tentativa, sendo que o critério de tentativa variou entre as condições. Na Linha de Base cada encontro caracterizava uma tentativa. $\mathrm{O}$ mesmo foi feito para a condição de $\mathrm{S}^{\mathrm{D}}$ no Treino de Discriminação. Para a condição de $S^{\Delta}$ no Treino, considerou-se como tentativa cada apresentação do estímulo (que se encerrava após $\mathrm{x}$ segundos sem ocorrência de encontro). Nas condições de Teste de Discriminação e de Generalização também se considerou cada apresentação do estímulo como uma tentativa. No Teste de Discriminação cada estímulo permanecia em vigor por 1 minuto, enquanto no Teste de Generalização cada estímulo permanecia em vigor por 2 minutos.

Para cada fase foram realizadas comparações intradupla e comparações entre duplas. Com as comparações intradupla pretendeu-se observar os possíveis efeitos diferenciados esperados para cada fase e entre cada estímulo $\left(\mathrm{S}^{\mathrm{D}}\right.$ e $\left.\mathrm{S}^{\Delta}\right)$. Com as comparações entre duplas esperou-se descrever tendências gerais observadas em cada fase, funcionando como replicações diretas.

\section{RESULTADOS}

A Figura 1 mostra a análise da taxa de PAs para as três primeiras fases do experimento. Observou-se que, apesar da variabilidade nas taxas, a ocorrência do PA foi estabelecida na Linha de Base. Para cinco das oito duplas (D3, D7, D8, D9 e D11) observou-se um aumento sistemático nas taxas de PA do começo ao final da fase. Para D11, observa-se um grande aumento da taxa no final da Linha de Base, entretanto, essa alta taxa não se manteve de maneira constante nas fases seguintes.

$\mathrm{Na}$ fase de Treino pode-se observar que, de maneira geral, a taxa durante as apresentações de $S^{\mathrm{D}}$ foram mais altas do que durante a apresentação do $\mathrm{S}^{\Delta}$.

Com a introdução da condição de $\mathrm{S}^{\Delta}$ pode-se observar, ainda, um aumento da taxa em $S^{\mathrm{D}}$ em relação à Linha de Base para seis das oito duplas (D3, D5, D7, D8, D9, D10), caracterizando assim o efeito de contraste comportamental. Para as duplas D3 e D10 o efeito de contraste foi mais evidente nas tentativas finais da fase de Treino. Para D6, embora se observe alguns pontos em que a taxa foi maior do que a obtida na linha de base, não houve um aumento sistemático da taxa de uma condição para outra. Para D11 se observa um grande aumento na taxa nas tentativas finais da Linha de Base, mas esse aumento não se manteve, nem se ampliou, durante o treino discriminativo.

Em relação à taxa em $\mathrm{S}^{\Delta}$ na fase de Treino, pode-se observar que, para todas as duplas, a taxa de PA na primeira apresentação desse estímulo foi 0 . Isso provavelmente se deveu a uma característica do procedimento, pois a exigência de inatividade nessa primeira apresentação era de 10 segundos sem ocorrência de encontros. Como essa exigência era menor do que o tempo médio para a ocorrência de um encontro, é possível que nessa primeira apresentação todas as duplas tenham alcançado o critério por acaso (o estímulo se encerrou antes que houvesse tempo suficiente para as peças se encontrarem). Embora tenha ocorrido um aumento das taxas nas tentativas seguintes em $\mathrm{S}^{\Delta}$, é observado um decréscimo sistemático ao longo da fase, para todas as duplas, exceto D10. A diferenciação entre as taxas de PA sob as duas condições de estímulos $\left(\mathrm{S}^{\mathrm{D}}\right.$ e $S^{\Delta}$ ) indicam o estabelecimento do controle discriminativo pelos diferentes estímulos apresentados.

Na fase de Teste de Discriminação observa-se que se manteve a maior taxa na presença do $S^{D}$ em relação ao $\mathrm{S}^{\Delta}$. As duplas D3, D7, D8, D10 e D11 apresentaram uma queda na taxa em $S^{D}$ em relação à fase anterior. Isso pode ser devido ao fato de que na fase de treino a presença do $S^{D}$ sinalizava a ocorrência de apenas um encontro, que era seguido do reforço e então se passava para o $S^{\Delta}$. Porém, na fase de Teste a duração de apresentação do $S^{D}$ obedecia a um critério de tempo e não de razão. Para as duplas D3, D5, D6, D7, D8 e D11 observou-se também um aumento, mesmo que transitório, da taxa em $\mathrm{S}^{\Delta}$. Apesar das flutuações nas taxas, para seis das oito duplas (D3, D5, D7, D8, D9 e D11) observou-se taxas bem diferenciadas entre os dois estímulos, evidenciando assim a manutenção da discriminação. Dessa forma, houve mais ocorrência de PA na condição associada ao reforço do que na condição associada à extinção. 

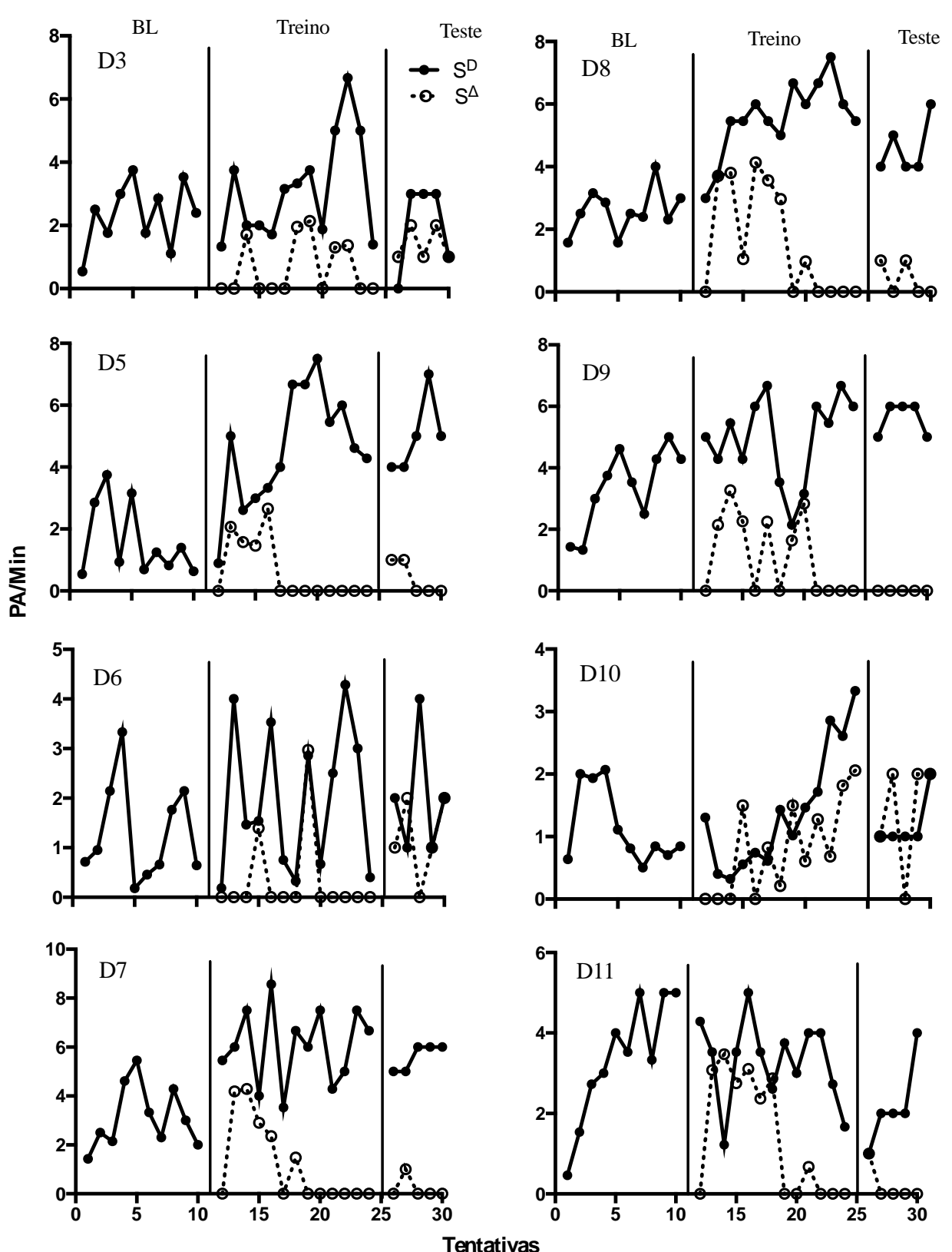

Figura 1. Taxa de produto agregado (PA/min), em cada tentativa ao longo das fases: Linha de Base, Treino de Discriminação e Teste de Discriminação. Cada gráfico se refere a uma dupla. Note que os valores do eixo y variam para cada dupla.

A dupla D10, além de não apresentar um decréscimo na taxa de PA na presença do $\mathrm{S}^{\Delta}$ durante o Treino, também não apresentou padrão de taxa diferenciada durante o Teste de Discriminação. Isso pode ter se devido ao fato de que essa dupla trabalhou a uma taxa muito baixa desde o início da sessão (suas taxas foram as menores dentre todas as duplas). Dessa forma, os critérios estabelecidos para o $\mathrm{S}^{\Delta}$ na fase de Treino podem ter sido alcançados por acaso (tempo médio entre as ocorrências dos encontros maiores do que 60 segundos, cumprindo assim o critério, sem necessariamente terem discriminado as condições).

Os dados no Teste de Generalização (Figura 2) não demonstraram sistematicidade. Apenas quatro das oito duplas (D3, D6, D8 e D11) apresentaram uma taxa de PA mais alta na presença do $S^{D}$, evidenciando o pico do gradiente de generalização previsto na literatura, porém, para nenhuma das duplas a taxa foi menor na presença desse estímulo, em relação aos demais. Não se observou o deslocamento do pico para qualquer uma das duplas.

Observou-se uma correlação entre a duração do Treino e o padrão de distribuição de respostas no Teste de Generalização. As duplas que apresentaram o pico de PA na presença do $S^{\mathrm{D}}$ durante o Teste de Generalização foram aquelas para as quais a fase de Treino teve duração maior do que a média (28'25'" para D3, 27'17' "para D6, 36'22'" para D8 e 28'13" para D11). D10 também obteve um tempo de Treino acima da média, embora não tenha demonstrado o padrão das demais duplas no Teste de Generalização. 

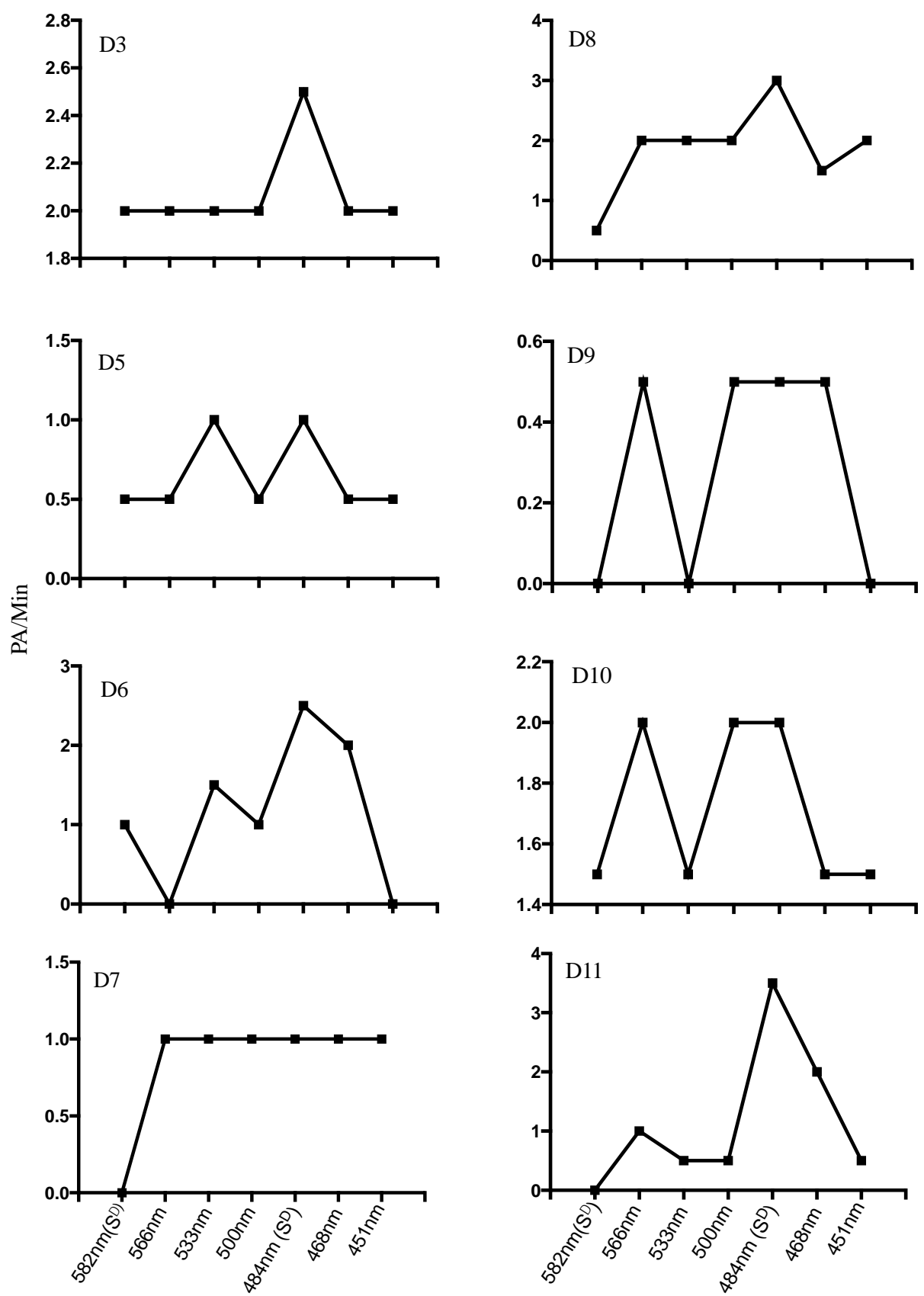

Figura 2. Taxa de produto agregado (PA/min) ao longo da fase de Teste de Generalização. Note que o eixo y varia para cada dupla.

Uma segunda análise realizada foi realizada em relação ao número de movimentos (Figura 3). O número de movimentos foi calculado também por tentativas, e assim como no cálculo das taxas, foram consideradas tentativas cada encontro na Linha de Base e na condição $S^{D}$ no Treino e cada apresentação de estímulo para a condição $\mathrm{S}^{\Delta}$ no Treino e para ambos os estímulos no Teste. Os movimentos na fase do Teste de Generalização não foram analisados.

Tal análise demonstrou que o número de movimentos se manteve constante durante as apresentações do $S^{\mathrm{D}}$, porém, variou consideravelmente durante as apresentações do $\mathrm{S}^{\Delta}$, sendo geralmente maior na presença desse estímulo. O aumento do número de movimentos em $\mathrm{S}^{\mathrm{D}}$ durante o Teste se deveu ao fato de que cada tentativa nessa fase tinha duração de um minuto, logo, ocorria mais de um encontro, diferente das fases antecedentes, em que cada encontro era considerado uma tentativa. A constância do número de movimentos em $\mathrm{S}^{\mathrm{D}}$ é um indício da estereotipia gerada pelo reforço, pois as duplas emitiam, em média, o mesmo número de movimentos até o encontro na presença do estímulo correlacionado ao reforço, mas variavam mais na presença do estímulo correlacionado à extinção. 

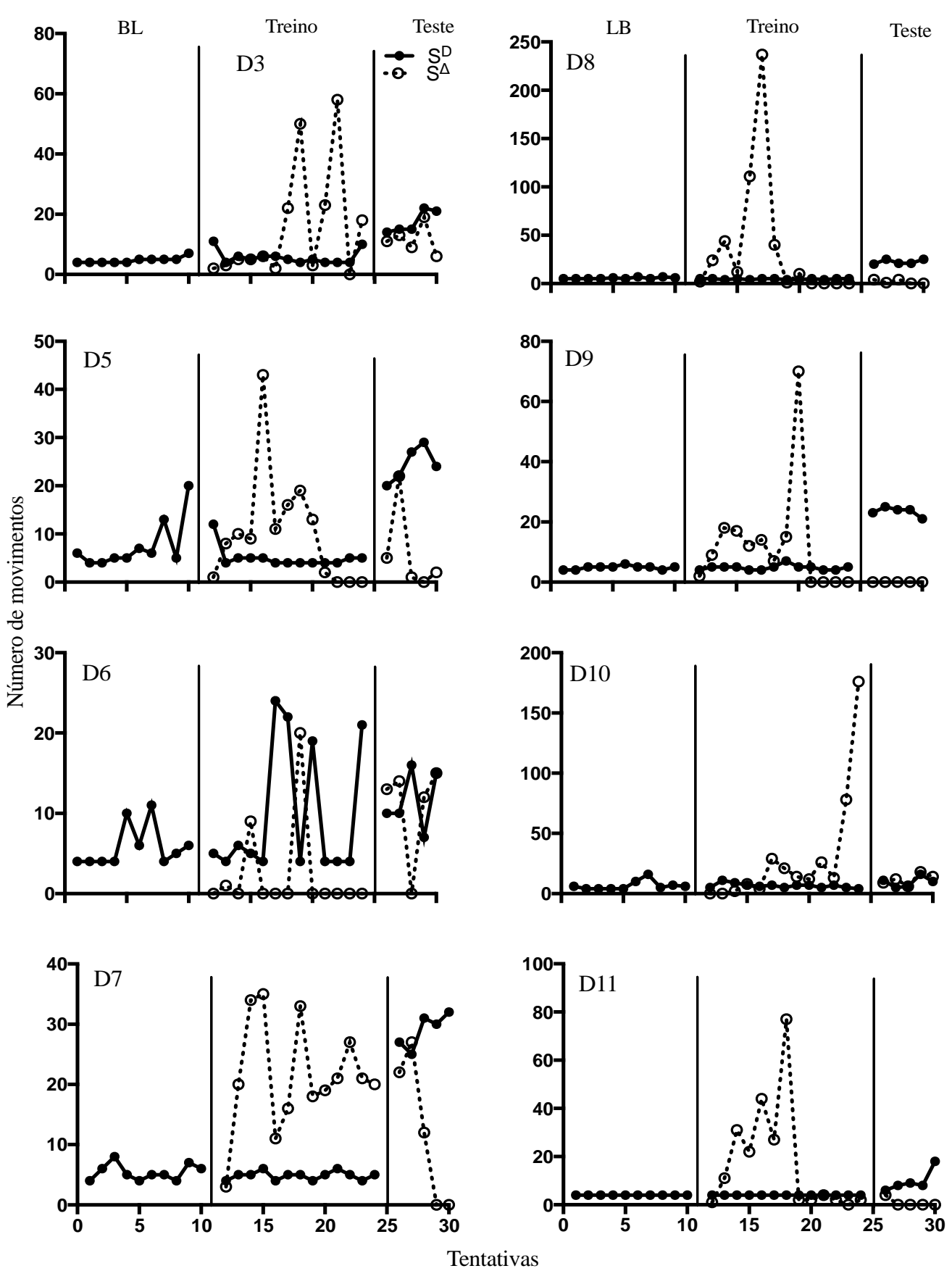

Figura 3. Número de movimentos, em cada tentativa, ao longo das condições experimentais (exceto Teste de Generalização). Cada gráfico se refere a uma dupla. Note que os valores do eixo y variam para cada dupla.

O cálculo do índice de dispersão (dados não mostrados) confirmou os resultados relativos ao número de movimentos, indicando o estabelecimento de estereotipia nas condições de reforço e variabilidade induzida pela extinção.

Entretanto, como exceção, a dupla D6 apresentou bastante variabilidade no número de movimentos, principalmente na presença do $\mathrm{S}^{\mathrm{D}}$, evidenciando assim que essa dupla não apresentou o padrão de estereotipia geralmente observado. Por outro lado, a dupla D10, embora não tenha apresentado indícios de discriminação
(Figura 1), emitiu, como as demais duplas, números regulares de movimentos na presença do $S^{\mathrm{D}}$.

De modo geral, essa análise permitiu perceber que diferentes padrões de comportamento foram estabelecidos pela contingência de extinção em vigor durante a apresentação do $S^{\Delta}$ na fase de treino: para algumas duplas se observou a extinção de qualquer tipo de movimento enquanto o estímulo estava em vigor (D5, D6, D8, D9); para outras duplas se observou a extinção da formação dos PAs e o desenvolvimento de outros padrões de comportamento, como por exemplo, o comportamento de "passear" pelo tabuleiro (D3, D7, D10 e D11). Dessa 
forma, há evidências de que, para algumas duplas o $\mathrm{S}^{\Delta}$ funcionou como uma contingência de extinção, enquanto para outras duplas funcionou como um DRO (tendo como reforço o retorno $S^{D}$ ).

\section{DISCUSSÃO}

O objetivo principal do presente estudo foi averiguar a possibilidade de estabelecimento de controle de estímulos para a ocorrência de Produtos Agregados, que envolvem comportamentos entrelaçados de mais de um indivíduo ao mesmo tempo, a partir de um procedimento simplificado.

O primeiro ponto a ser observado é que a utilização do procedimento se mostrou eficiente em demonstrar o estabelecimento da ocorrência do PA sem envolver modelagem de comportamentos individuais e sem utilizar consequências diferenciais para cada indivíduo, assim como foi observado por Vasconcelos \& Todorov (2015). A formação de PAs ao longo de toda a sessão experimental demonstrou, portanto, a viabilidade da utilização de um procedimento mais simples para investigação de fenômenos que ocorrem em um terceiro nível de seleção.

Apesar da variabilidade observada nos dados, há indícios de que o controle discriminativo foi estabelecido em função das taxas diferenciais de PA na presença de ambos os estímulos. O padrão observado durante o Teste de Discriminação é um indicador do controle discriminativo exercido por cada estímulo, muito embora não se observe sistematicidade para todas as duplas durante o Teste de Generalização. Os indicativos do estabelecimento de discriminação nesse estudo confirmam e estendem para outra tarefa os resultados de Vieira (2010), que também observou controle pelos estímulos antecedentes na formação de Produtos Agregados. Assim como no presente estudo, Vieira (2010) também não observou sistematicidade no teste de generalização.

Nos resultados do presente estudo pode-se observar também que, embora tenham ocorrido taxas diferenciadas de produto agregado entre os estímulos durante a fase de Teste de Discriminação, ocorreu também uma pequena diminuição na taxa nesta fase, em relação ao Treino, para algumas duplas (D7, D8, D9, D10 e D11). Essa diferença pode se dever à alteração na duração dos estímulos. No treino, a mudança de $S^{D}$ para $S^{\Delta}$ se dava a cada encontro e havia uma contingência de atraso da duração de $\mathrm{S}^{\Delta}$ contingente a cada ocorrência de PA. Na fase de Teste foi introduzida uma modificação na contingência associada à apresentação de cada estímulo, estando esses em vigor por intervalos fixos de tempo (1 min para cada estímulo). Dessa forma, cada encontro ocorrido na presença do $S^{\mathrm{D}}$ não sinalizava mais o início do $\mathrm{S}^{\Delta}$. Essa mudança na contingência de apresentação dos estímulos de uma fase para outra pode ser apontada como um fator que pode ter influenciado no padrão observado.

Uma possível explicação para a falta de sistematicidade dos dados de generalização no presente trabalho pode ter sido, então, essa mudança na duração dos estímulos de uma fase para a outra, atuando como uma variável estranha no estabelecimento da discriminação.
Outro fator que pode também ter afetado o estabelecimento da discriminação, foi a alternância entre estímulos. Por exemplo, durante o treino cada encontro ocorrido na presença do $\mathrm{S}^{\mathrm{D}}$ sinalizava não apenas a apresentação do reforço, como também a mudança para a condição de $\mathrm{S}^{\Delta}$. Assim, a presença de um estímulo sinalizava, necessariamente, a apresentação do outro estímulo em seguida, uma vez que os estímulos eram apresentados sucessivamente e não de maneira aleatória. No Teste de Generalização, a maneira e a ordem na qual as cores foram apresentadas também podem ter contribuído para a distribuição das respostas, tendo em vista que quando o $S^{D}$ era apresentado nessa fase a dupla já estava há muitos minutos em extinção. Outra possível explicação pode ter sido a extensão da sessão, de forma que as fases de treino e teste de discriminação podem não ter sido longas o suficiente para gerar um padrão bem estabelecido de discriminação.

Foi possível observar o efeito de contraste comportamental quando ocorreu a introdução do $\mathrm{S}^{\Delta}$ na fase de Treino, confirmando a literatura sobre treinos de discriminação (Reynolds, 1961a e 1961b). Os dados estão de acordo com aqueles demonstrados por Reynolds (1961a), que indicam o efeito contraste relacionado à ausência de reforço no componente variável.

O contraste comportamental observado neste trabalho pode ser considerado o que Killeen (2014) denomina de contraste transitório, por ser observado já na fase inicial do treino. O autor afirma, ainda, que a duração dos componentes pode ter efeito sobre esse tipo de contraste. Isso pode explicar, também, algumas das diferenças observadas nas taxas de produto agregado entre as fases de Treino e Teste de Discriminação. Durante a fase de Treino, a duração de cada $S^{D}$ era o equivalente à duração para ocorrência do encontro. Porém, a a duração do $\mathrm{S}^{\Delta}$ era função do tempo exigido sem ocorrência do PA (sendo o intervalo atrasado a cada ocorrência deste), e sendo essa exigência de 1 minuto nas três últimas apresentações do estímulo durante a fase de treino. Com exceção da dupla D10, para todas as outras duplas não houve encontro nas últimas apresentações do $\mathrm{S}^{\Delta}$; dessa forma, a duração do estímulo foi de 1 minuto, de acordo com o estabelecido pelo critério. $\mathrm{Na}$ fase de Teste, cada estímulo passou a ficar em vigor pelo intervalo fixo de 1 minuto, logo, a duração do $\mathrm{S}^{\mathrm{D}}$ passou a ser maior do que era na fase de Treino (em que, em geral, cada PA ocorria em um tempo médio menor do que 1 minuto), porém, a duração do $\mathrm{S}^{\Delta}$ se manteve a mesma das tentativas finais durante o treino. Assim, houve uma mudança na duração de um dos componentes (componente correlacionado ao reforço), mas não houve mudança da duração do segundo componente (componente correlacionado à extinção). Segundo Killeen (2014), o aumento da duração do componente correlacionado ao reforço, enquanto o segundo componente se manteve constante, pode ter como um dos efeitos a diminuição do contraste, tal como observado no presente trabalho, em função da queda na taxa de PA durante o Teste de Discriminação (e.g., Shimp \& Wheatley, 1971; Todorov, 1972). 
Os dados em relação aos números de movimentos e índice de dispersão, também confirmaram os efeitos, já observados no nível operante, de estereotipia gerada pelo reforçamento e variabilidade gerada pela extinção. Esses efeitos já haviam sido observados no experimento de Vasconcelos \& Todorov (2015).

De maneira geral, o presente estudo demonstrou a possibilidade de se trabalhar com um procedimento simplificado que atende às definições do conceito de metacontingência, além de dar indícios de que, assim como no nível operante, o controle exercido pelos estímulos antecedentes parece ter relevância também quando se fala em um terceiro nível de seleção (Houmanfar \& Rodrigues, 2006). Faz-se importante, então, avanços nas investigações sobre os processos básicos envolvidos no terceiro nível de seleção, a fim de se observar se os princípios estabelecidos pelos estudos do comportamento operante também se aplicam a este nível.

\section{REFERÊNCIAS}

Azrin, N. H., \& Lindsley, O. R. (1956). Reinforcement of cooperation between children. Journal of Abnormal and Social Psychology, 52, 100-102.

Dinsmoor, J. A. (1950). A quantitative comparison of the discriminative and reinforcing functions of a stimulus. Journal of Experimental Psychology,40, 458-472.

Glenn, S. S., \& Malott, M. (2004). Complexity and selection: Implications for organizational change. Behavior and Social Issues, 13, 89-106.

Hake, D. F., Olvera, D., \& Bell, J. C. (1975). Switching from competition to sharing or cooperation at large response requirements: Competition requires more responding. Journal of the Experimental Analysis of Behavior, 24, 343-354.

Honig, W. K., \& Urcuioli, P. J. (1981). The legacy of Guttman and Kalish (1956): 25 years of research on stimulus generalization. Journal of the Experimental Analysis of Behavior, 36, 405-445.

Houmanfar, R., \& Rodrigues, N. J. (2006). The metacontingency and the behavior contingency: Points of contact and departure. Behavior and Social Issues, 15, 13-30.

Killeen, P. R. (2014). A theory of behavioral constrast. Journal of the Experimental Analysis of Behavior, 102, 363-390.

McSweeney, F. K., Ettinger, R. H., \& Norman, W. D. (1981). Three versions of the additive theories of behavioral contrast. Journal of the Experimental Analysis of Behavior, 36, 285-297.

Morse, W. H., \& Skinner, B. F. (1958). Some factors involved in the stimulus control of operant behavior. Journal of the Experimental Analysis of Behavior, 1(1), 103-107.

Pereira, J. M. C. (2008). Investigação experimental de metacontingências: separação do produto agregado $e$ da consequência individual. Dissertação de mestrado. Pontifícia Universidade Católica de São Paulo, São Paulo, SP, Brasil.
Reynolds, G. S. (1961a). Behavioral contrast. Journal of the Experimental Analysis of Behavior, 4, 57-71.

Reynolds, G. S. (1961b). Contrast, generalization and the process of discriminations. Journal of the Experimental Analysis of Behavior, 4, 289-294.

Schimitt, D. R. (1976). Some conditions affecting the choice to cooperate or compete. Journal of the Experimental Analysis of Behavior, 25, 165-178.

Schimitt, D. R., \& Marwell, G. (1968). Stimulus control in the experimental study of cooperation. Journal of the Experimental Analysis of Behavior, 11, 571-574.

Schimitt, D. R., \& Marwell, G. (1971a). Avoidance of risk as a determinant of cooperation. Journal of the Experimental Analysis of Behavior, 16, 367-374.

Schimitt, D. R., \& Marwell, G. (1971b). Taking and the disruption of cooperation. Journal of the Experimental Analysis of Behavior, 15, 405-412.

Sério, T. M. A. P., Andery, M. A., Gioia, P. S., \& Micheletto, N. (2002). Os conceitos de discriminação e generalização. In: T. M. A. P. Sério, M. A. Andery, P. S. Gioia, \& N. Micheletto. In Controle de estímulos e comportamento operante (pp. 7-24). São Paulo: EDUC.

Shimp, C. P., \& Wheatley, K. L. (1971). Matching to relative reinforcement frequency in multiple schedules with a short component duration. Journal of the Experimental Analysis of Behavior, 15, 205-210.

Sidman, M. (2008). Reflections on stimulus control. The Behavior Analyst, 31(2), 127-135.

Skinner, B. F. (2003). Ciência e comportamento humano (11 ${ }^{\mathrm{a}}$ ed.). Trad. de J. C. Todorov e R. Azzi. São Paulo: Martins Fontes. (Obra original publicada em 1953).

Soares, P. F., Cabral, P. A., Leite, F. L., \& Tourinho, E. Z. (2014). Efeitos de consequências culturais sobre a seleção e manutenção de duas práticas culturais alternadas. Revista Brasileira de Análise do Comportamento, 8, 37-46.

Terrace, H. S. (1966). Behavioral contrast and the peak shift: Effects of extended discrimination training. Journal of the Experimental Analysis of Behavior, 9, 613-617.

Todorov, J. C. (1972). Component duration and relative response rates in multiple schedule. Journal of the Experimental Analysis of Behavior, 17, 45-49.

Todorov, J. C. (2002). A evolução do conceito de operante. Psicologia: Teoria e Pesquisa, 18, 123-127.

Todorov, J. C. (2012a). Metacontingências e a análise comportamental de práticas culturais. Clínica \& Cultura, 1, 36-45.

Todorov, J. C. (2012b). Contingências de seleção cultural. Revista Brasileira de Análise do Comportamento, 8, 49-59.

Todorov, J. C. (2013). Conservation and transformation of cultural practices through contingencies and metacontingencies. Behavior and Social Issues, 22, 64 73.

Todorov, J. C., \& Vianney, J. B. (2013). Xadrez (Versão 3.4.5) [Programa de computador]. Brasília, DF: Brasil.

Vasconcelos, I. G., \& Todorov, J. C. (2015). Experimental analysis of the behavior of persons in groups: Selection 
of an aggregate product in a metacontingency. Behavior and Social Issues, 24, 111-125.

Vichi, C. (2004). Igualdade ou desigualdade em pequeno grupo: um análogo experimental de manipulação de uma prática cultural. Dissertação de mestrado. Pontifícia Universidade Católica de São Paulo, São Paulo, SP, Brasil.

Vieira, M.C. (2010) Condições antecedentes participam de metacontingências? Dissertação de mestrado. São Paulo: Pontifícia Universidade Católica de São Paulo.

Submetido em 15/06/2016

Aceito em 06/03/2017 